\title{
Toward Controlling the Solid State Valence Tautomeric Interconversion Character by Solvation
}

Marcos A. Ribeiro ${ }^{1}$, Daniel E. Stasiw ${ }^{2}$, Philip Pattison ${ }^{3}$, Paul R. Raithby ${ }^{4}$, David A. Shultz ${ }^{2}$ and Carlos B. Pinheiro ${ }^{1 *}$.

1- Departamento de Física, Universidade Federal de Minas Gerais, Belo Horizonte, Brazil.

2- Department of Chemistry, North Carolina State University, Raleigh, USA,

3- Swiss-Norwegian Beam Line, ESRF, Grenoble Cedex, France

4- Department of Chemistry, University of Bath, Bath, United Kingdom.

"E-mail: cbpinheiro@ufmg.br

KEYWORDS Valence Tautomers, cobalt, dioxolene.

Supporting Information 
Table S1: Measurements temperatures

\begin{tabular}{|c|c|c|c|c|c|c|c|}
\hline Sample & $\begin{array}{c}\text { 1BZ } \\
\text { Cooling }\end{array}$ & $\begin{array}{c}\text { 1BZ } \\
\text { Heating }\end{array}$ & $\begin{array}{c}\text { 1BZ } \\
\text { Heating }\end{array}$ & $\begin{array}{c}\text { 1TL } \\
\text { Heating }\end{array}$ & $\begin{array}{c}\text { 1TL } \\
\text { Cooling }\end{array}$ & $\begin{array}{c}\text { 2BZ } \\
\text { Cooling }\end{array}$ & $\begin{array}{c}2 \mathrm{TI} \\
\text { Cooling }\end{array}$ \\
\hline \multirow{11}{*}{ 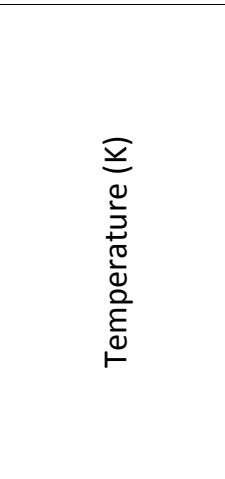 } & 110 & 110 & 97 & 100 & 100 & 123 & 123 \\
\hline & 120 & 125 & 100 & 180 & 120 & 153 & 153 \\
\hline & 140 & 140 & 120 & 210 & 150 & 183 & 183 \\
\hline & 150 & 155 & 140 & 250 & 180 & 213 & 213 \\
\hline & 170 & 170 & 150 & & 210 & 243 & 243 \\
\hline & 200 & 185 & 160 & & 240 & 273 & 273 \\
\hline & 230 & 200 & 180 & & 270 & & \\
\hline & 260 & 215 & 200 & & & & \\
\hline & 280 & 250 & 220 & & & & \\
\hline & & 290 & 240 & & & & \\
\hline & & & 290 & & & & \\
\hline Diffractometer & $\begin{array}{c}\text { Gemini } \\
\text { Bath } \\
\text { UK }\end{array}$ & $\begin{array}{c}\text { Gemini } \\
\text { Bath } \\
\text { UK }\end{array}$ & $\begin{array}{c}\text { Gemini } \\
\text { LabCri } \\
\text { Brazil }\end{array}$ & $\begin{array}{l}\text { SNBL } \\
\text { ESRF }\end{array}$ & $\begin{array}{c}\text { Gemini } \\
\text { LabCri } \\
\text { Brazil }\end{array}$ & $\begin{array}{c}\text { Gemini } \\
\text { Bath } \\
\text { UK }\end{array}$ & $\begin{array}{c}\text { Gemini } \\
\text { Bath } \\
\text { UK }\end{array}$ \\
\hline
\end{tabular}


Table S2: Sample characteristics, measurements and refinements data of $\left[\mathrm{Co}(\text { diox })_{2}\left(4-\mathrm{NO}_{2}-\mathrm{py}\right)_{2}\right]$.benzene $(1 \mathrm{BZ})$ and $\left[\mathrm{Co}(\text { diox })_{2}\left(4-\mathrm{NO}_{2} \text {-py }\right)_{2}\right]$. toluene $(1 \mathrm{TL})$ solvate crystals at different temperatures. diox $=3,5$-di-tbutylsemiquinonate ( $\left.S Q^{--}\right)$and/or 3,5-di-t-butylcatecholate $\left(\mathrm{Cat}^{2-}\right)$.

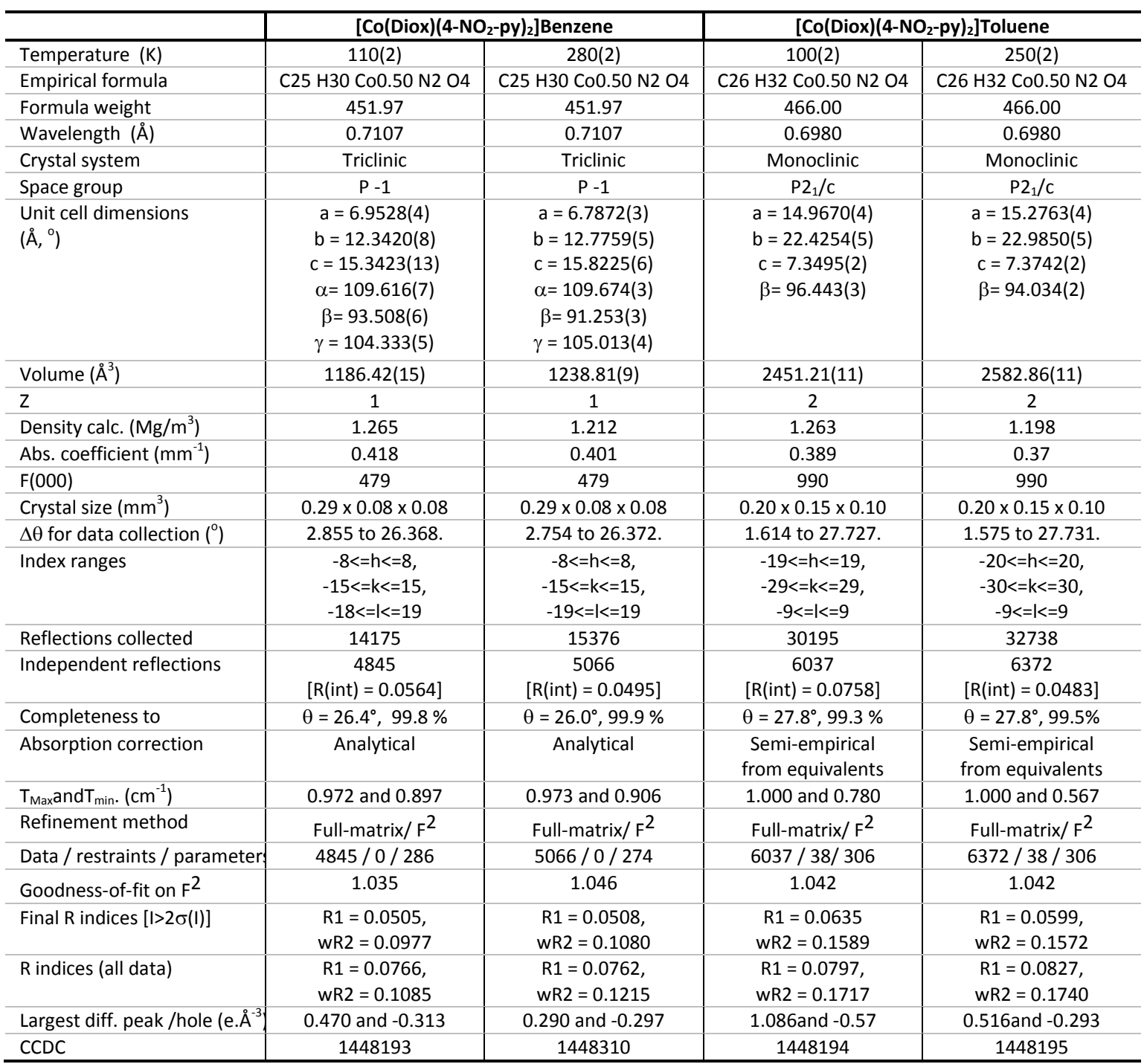


Table S3: Sample characteristics, measurements and refinements data of $\left[\mathrm{Co}(\text { diox })_{2}(4-\mathrm{CN}-\mathrm{py})_{2}\right]$. benzene (2BZ) and $\left[\mathrm{Co}(\text { diox })_{2}(4-\mathrm{CN} \text {-py) })_{2}\right.$.toluene $(\mathbf{2 T L})$ solvate crystals at different temperatures. diox $=3,5$-di-tbutylsemiquinonate $\left(S Q^{-}\right)$and/or 3,5-di-t-butylcatecholate $\left(\mathrm{Cat}^{2-}\right)$.

\begin{tabular}{|c|c|c|c|c|}
\hline & \multicolumn{2}{|c|}{ [Co(Diox)(4-CN-py) 2 Benzene } & \multicolumn{2}{|c|}{ [Co(Diox)(4-CN-py) $\left.)_{2}\right]$ Toluene } \\
\hline Temperature $(\mathrm{K})$ & $123(2)$ & $273(2)$ & $123(2)$ & $273(2)$ \\
\hline Empirical formula & $\mathrm{C} 26 \mathrm{H} 30 \mathrm{CoO} .50 \mathrm{~N} 2 \mathrm{O} 2$ & $\mathrm{C} 26 \mathrm{H} 30 \mathrm{Co} 0.50 \mathrm{~N} 2 \mathrm{O} 2$ & $\mathrm{C} 27 \mathrm{H} 32 \mathrm{CoO} .50 \mathrm{~N} 2 \mathrm{O} 2$ & $\mathrm{C} 27 \mathrm{H} 32 \mathrm{Co} 0.50 \mathrm{~N} 2 \mathrm{O} 2$ \\
\hline Formula weight & 431.98 & 431.98 & 446.01 & 446.01 \\
\hline Wavelength $(\AA)$ & 1.5418 & 1.5418 & 1.5418 & 1.5418 \\
\hline Crystal system & Monoclinic & Monoclinic & Monoclinic & Monoclinic \\
\hline Space group & $\mathrm{P} 22_{1} / \mathrm{c}$ & $\mathrm{P} 2{ }_{1} / \mathrm{c}$ & $\mathrm{P} 22_{1} / \mathrm{c}$ & $\mathrm{P} 2{ }_{1} / \mathrm{c}$ \\
\hline $\begin{array}{l}\text { Unit cell dimensions } \\
\left(\AA \AA^{\circ}\right)\end{array}$ & $\begin{array}{c}a=15.017(5) \\
b=22.095(5) \\
c=7.316(5) \\
\beta=97.392(5)\end{array}$ & $\begin{array}{c}a=15.3229(8) \\
b=22.6769(12) \\
c=7.2956(4) \\
\beta=95.104(5)\end{array}$ & $\begin{array}{c}a=15.3358(3) \\
b=22.0224(4) \\
c=7.38730(10) \\
\beta=91.363(2)\end{array}$ & $\begin{array}{c}a=15.4001(5) \\
b=22.8629(8) \\
c=7.3621(3) \\
\beta=93.527(3)\end{array}$ \\
\hline Volume $\left(\AA^{3}\right)$ & 2407.3(19) & $2525.0(2)$ & $2494.22(7)$ & $2587.22(16)$ \\
\hline Z & 2 & 2 & 2 & 2 \\
\hline Density $\mathrm{Mg} / \mathrm{m}^{3}$ (calculated) & 1.192 & 1.136 & 1.188 & 1.145 \\
\hline Abs. coefficient $\left(\mathrm{mm}^{-1}\right)$ & 3.172 & 3.029 & 3.051 & 2.954 \\
\hline$F(000)$ & 918 & 918 & 950 & 950 \\
\hline Crystal size $\left(\mathrm{mm}^{3}\right)$ & $0.31 \times 0.05 \times 0.04$ & $0.8 \times 0.12 \times 0.09$ & $0.58 \times 0.20 \times 0.16$ & $0.63 \times 0.20 \times 0.15$ \\
\hline$\Delta \theta$ for data collection $\left({ }^{\circ}\right)$ & 2.967 to 62.462 & 2.895 to 62.570 & 2.882 to 66.860 & 2.875 to 66.751 \\
\hline Index ranges & $\begin{array}{c}-17<=\mathrm{h}<=14, \\
-25<=\mathrm{k}<=25, \\
-7<=\mid<=8\end{array}$ & $\begin{array}{c}-15<=\mathrm{h}<=17, \\
-25<=\mathrm{k}<=26, \\
-8<=\mathrm{<}<=7\end{array}$ & $\begin{array}{c}-17<=h<=18, \\
-26<=k<=26, \\
-8<=\mid<=6\end{array}$ & $\begin{array}{c}-18<=\mathrm{h}<=18, \\
-26<=\mathrm{k}<=27, \\
-8<=\mathrm{k}<=8\end{array}$ \\
\hline Reflections collected & 14040 & 14706 & 14109 & 16011 \\
\hline Independent reflections & $\begin{array}{c}3842 \\
{[R \text { (int) }=0.0342]}\end{array}$ & $\begin{array}{c}4004 \\
{[R(\text { int })=0.0626]}\end{array}$ & $\begin{array}{c}4405 \\
{[R(\text { int })=0.0368]}\end{array}$ & $\begin{array}{c}4588 \\
{[R(\text { int })=0.0448]}\end{array}$ \\
\hline Completeness to & $\theta=62.5^{\circ}, 99.6 \%$ & $\theta=63.0^{\circ}, 97.7 \%$ & $\theta=67.8^{\circ}, 97.6 \%$ & $\theta=67.8^{\circ}, 98.2 \%$ \\
\hline Absorption correction & Analytical & Analytical & Analytical & Analytical \\
\hline $\mathrm{T}_{\text {Max }}$ and $\mathrm{T}_{\min } \cdot\left(\mathrm{cm}^{-1}\right)$ & 0.881 and 0.601 & 0.767 and 0.310 & 0.656 and 0.360 & 0.671 and 0.320 \\
\hline Refinement method & Full-matrix/ $\mathrm{F}^{2}$ & Full-matrix/ $\mathrm{F}^{2}$ & Full-matrix/ $\mathrm{F}^{2}$ & Full-matrix/ $\mathrm{F}^{2}$ \\
\hline Data / restraints / parameters & 3842 / 36 / 283 & 4004 / 36 / 271 & 4405 / 45 / 294 & 4588 / $161 / 311$ \\
\hline Goodness-of-fit on $\mathrm{F}^{2}$ & 1.018 & 1.086 & 1.051 & 1.03 \\
\hline Final $R$ indices $[1>2 \sigma(I)]$ & $\begin{array}{l}R 1=0.0344, \\
w R 2=0.0807\end{array}$ & $\begin{array}{c}R 1=0.0522, \\
w R 2=0.1263\end{array}$ & $\begin{array}{l}R 1=0.0460, \\
w R 2=0.1121\end{array}$ & $\begin{array}{l}R 1=0.0584 \\
w R 2=0.1657\end{array}$ \\
\hline $\mathrm{R}$ indices (all data) & $\begin{array}{c}R 1=0.0423 \\
w R 2=0.0857\end{array}$ & $\begin{array}{l}\mathrm{R} 1=0.0864 \\
\mathrm{wR} 2=0.1491\end{array}$ & $\begin{array}{l}R 1=0.0577 \\
w R 2=0.1198\end{array}$ & $\begin{aligned} R 1 & =0.0780, \\
w R 2 & =0.1842\end{aligned}$ \\
\hline Largest diff. peak /hole $\left(e . \AA^{-3}\right)$ & 0.239 and -0.288 & 0.215 and -0.236 & 0.512 and -0.270 & 0.405 and -0.278 \\
\hline CCDC & 1448196 & 1448197 & 1448198 & 1448311 \\
\hline
\end{tabular}


Table S4: Intra and intermolecular hydrogen bonding in [Co(diox $\left.)_{2}\left(4-\mathrm{NO}_{2}-\mathrm{py}\right)_{2}\right]$. Benzene (1BZ) solvate crystal at different temperatures. $C_{a r}$ stands for aromatic carbon and $\mathrm{C}_{\mathrm{s}}$ for carbon of solvent molecules.

\begin{tabular}{|c|c|c|c|c|c|c|c|}
\hline \multicolumn{8}{|c|}{$1 B Z$} \\
\hline & Temp (K) & Type & $D-H \ldots A$ & $d(D-H)(\AA)$ & $d(H \ldots A)(\AA)$ & $d(D \ldots A)(\AA)$ & $(\mathrm{DHA})\left(^{\circ}\right)$ \\
\hline \multirow{5}{*}{ 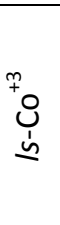 } & \multirow{5}{*}{110} & $\mathrm{C}_{\mathrm{ar}}-\mathrm{H}^{\cdots} \mathrm{O}_{\text {diox }}$ & $\mathrm{C} 16-\mathrm{H} 16 \ldots \mathrm{O} 2^{i}$ & 0.95 & 2.27 & $3.122(3)$ & 148.0 \\
\hline & & $\mathrm{C}_{\mathrm{ar}}-\mathrm{H}^{\cdots} \mathrm{O}$ ONO & $\mathrm{C} 18-\mathrm{H} 18 \ldots . \mathrm{O} 3^{\mathrm{ii}}$ & 0.95 & 2.47 & $3.293(3)$ & 144.8 \\
\hline & & $\mathrm{C}_{\mathrm{s}}-\mathrm{H} \cdots \mathrm{ONO}$ & $\mathrm{C} 22-\mathrm{H} 22 \ldots \mathrm{OO} 4^{\mathrm{iii}}$ & 0.95 & 2.66 & $3.308(3)$ & 125.8 \\
\hline & & $\mathrm{C}_{\mathrm{ar}}-\mathrm{H}^{\cdots} \mathrm{O}_{\text {diox }}$ Intra & C15-H15...O1 & 0.95 & 2.35 & $2.840(3)$ & 111.7 \\
\hline & & $\mathrm{C}_{\mathrm{ar}}-\mathrm{H}^{\cdots \cdots} \mathrm{O}_{\text {diox }}$ Intra & $\mathrm{C} 19-\mathrm{H} 19 . . . \mathrm{O} 1^{\mathrm{iv}}$ & 0.95 & 2.35 & $2.824(3)$ & 110.4 \\
\hline \multirow{5}{*}{ 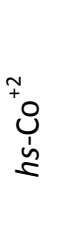 } & \multirow{5}{*}{280} & $\mathrm{C}_{\mathrm{ar}}-\mathrm{H}^{\cdots} \mathrm{O}_{\text {diox }}$ & $\mathrm{C} 16-\mathrm{H} 16 \ldots \mathrm{O} 2^{\mathrm{i}}$ & 0.93 & 2.35 & $3.124(3)$ & 140.0 \\
\hline & & $\mathrm{C}_{\mathrm{ar}}-\mathrm{H}^{\cdots} \mathrm{O}_{\text {diox }}$ & $\mathrm{C} 18-\mathrm{H} 18 \ldots . \mathrm{O} 3^{\mathrm{ii}}$ & 0.93 & 2.50 & $3.323(3)$ & 147.8 \\
\hline & & $\mathrm{C}_{\mathrm{s}}-\mathrm{H}^{\prime} \mathrm{O} \mathrm{ONO}$ & $\mathrm{C} 22-\mathrm{H} 22 \ldots \mathrm{O} 4^{\mathrm{iii}}$ & 0.93 & 2.64 & $3.333(3)$ & 131.6 \\
\hline & & $\mathrm{C}_{\mathrm{ar}}-\mathrm{H}^{\cdots \cdots} \mathrm{O}_{\text {diox }}$ Intra & $\mathrm{C} 15-\mathrm{H} 15 \ldots \mathrm{O} 1$ & 0.93 & 2.61 & $3.136(3)$ & 116.7 \\
\hline & & $\mathrm{C}_{\mathrm{ar}}-\mathrm{H}^{\cdots} \mathrm{O}_{\text {diox }}$ Intra & $\mathrm{C} 19-\mathrm{H} 19 . . . \mathrm{O} 1^{\text {iv }}$ & 0.93 & 2.49 & $3.051(3)$ & 118.9 \\
\hline
\end{tabular}

$P-1$ space group symmetry operation codes: $i=\mathrm{x}-1, \mathrm{y}, \mathrm{z} ; i i=-\mathrm{x}+1,-\mathrm{y}+1,-\mathrm{z}+1$;

$i i i=\mathrm{x}+1, \mathrm{y}, \mathrm{z}, i v=-\mathrm{x}+1,-\mathrm{y},-\mathrm{z}+1$

Table S5: Intra and intermolecular hydrogen bonding in [Co(diox $\left.)_{2}\left(4-\mathrm{NO}_{2}-\mathrm{py}\right)_{2}\right]$.Toluene (1TL) solvate crystal at different temperatures.

\begin{tabular}{|c|c|c|c|c|c|c|c|}
\hline $1 T L$ & & & & & & & \\
\hline Temp & Temp (K) & Type & D-H...A & $d(D-H)$ & $d(H \ldots A)$ & $d(D \ldots A)$ & $<(\mathrm{DHA})$ \\
\hline \multirow{4}{*}{$\begin{array}{l}+0 \\
\underbrace{+}_{0} \\
\vdots \\
\vdots\end{array}$} & \multirow{4}{*}{100} & $\mathrm{C}_{\mathrm{ar}}-\mathrm{H}^{\cdots} \mathrm{O}_{\text {diox }}$ & $\mathrm{C} 16-\mathrm{H} 16 \ldots \mathrm{O} 2^{i}$ & 0.95 & 2.42 & $3.319(3)$ & 157.9 \\
\hline & & $\mathrm{C}_{\mathrm{ar}}-\mathrm{H}^{\cdots} \mathrm{O} \mathrm{ONO}$ & $\mathrm{C} 18-\mathrm{H} 18 \ldots \mathrm{O} 3^{\mathrm{ii}}$ & 0.95 & 2.47 & $3.379(3)$ & 160.6 \\
\hline & & $\mathrm{C}_{\mathrm{ar}}-\mathrm{H}^{\prime \cdots} \mathrm{O}_{\text {diox }}$ Intra & C15-H15...O1 & 0.95 & 2.33 & $2.837(3)$ & 111.6 \\
\hline & & $\mathrm{C}_{\mathrm{ar}}-\mathrm{H}^{\cdots} \mathrm{O}_{\text {diox }}$ Intra & $\mathrm{C} 19-\mathrm{H} 19 . . . \mathrm{O} 1^{\mathrm{iii}}$ & 0.95 & 2.40 & $2.850(3)$ & 108.7 \\
\hline & & $\mathrm{C}_{\mathrm{s}}-\mathrm{H} \cdots \mathrm{ONO}$ & $\mathrm{C} 22-\mathrm{H} 22 \cdots \mathrm{O} 4^{\mathrm{iv}}$ & 0.95 & 2.77 & $3.418(3)$ & 126.2 \\
\hline \multirow{4}{*}{\begin{tabular}{l}
+1 \\
\multirow{1}{+}{0} \\
$\vdots$ \\
$\Sigma$
\end{tabular}} & \multirow{4}{*}{250} & $\mathrm{C}_{\mathrm{ar}}-\mathrm{H}^{\cdots} \mathrm{O}_{\text {diox }}$ & $\mathrm{C} 16-\mathrm{H} 16 \ldots \mathrm{O} 2^{i}$ & 0.94 & 2.46 & $3.337(4)$ & 155.1 \\
\hline & & $\mathrm{C}_{\mathrm{ar}}-\mathrm{H}^{\cdots} \mathrm{ONO}$ & $\mathrm{C} 18-\mathrm{H} 18 \ldots . \mathrm{O}^{\mathrm{ii}}$ & 0.94 & 2.50 & $3.384(4)$ & 155.8 \\
\hline & & $\mathrm{C}_{\mathrm{ar}}-\mathrm{H}^{\cdots} \mathrm{O}_{\text {diox }}$ Intra & $\mathrm{C} 15-\mathrm{H} 15 \ldots \mathrm{O} 1$ & 0.94 & 2.50 & $3.051(3)$ & 117.6 \\
\hline & & $\mathrm{C}_{\mathrm{ar}}-\mathrm{H}^{\cdots} \mathrm{O}_{\text {diox }}$ Intra & $\mathrm{C} 19-\mathrm{H} 19 . . . \mathrm{O}^{\mathrm{iv}}$ & 0.94 & 2.56 & $3.084(3)$ & 115.3 \\
\hline & & $\mathrm{C}_{\mathrm{s}}-\mathrm{H}^{\prime \cdots} \mathrm{ONO}$ & $\mathrm{C} 22-\mathrm{H} 22 \cdots \mathrm{O} 4^{\mathrm{iv}}$ & 0.94 & 2.85 & $3.554(5)$ & 132.8 \\
\hline
\end{tabular}

$\mathrm{P} 2{ }_{1} / \mathrm{c}$ space group $\mathrm{i}=\mathrm{x}, \mathrm{y}, \mathrm{z}-1$; $\mathrm{ii}=\mathrm{x},-\mathrm{y}+1 / 2, \mathrm{z}+1 / 2 ; \mathrm{iii}=-\mathrm{x}+2,-\mathrm{y}+1,-\mathrm{z}+1$; $\mathrm{iv}=-x+2,-y+1,-z+1$; iv $=x, y, z+1$

Table S6: Intra and intermolecular hydrogen bonding in $\left[\mathrm{Co}(\text { diox })_{2}(4-\mathrm{CN}-\mathrm{py})_{2}\right]$. Toluene (2TL) solvate crystal at different temperatures.

\begin{tabular}{|c|c|c|c|c|c|c|c|}
\hline $2 T L$ & & & & & & & \\
\hline Temp & Temp (K) & Type & D-H...A & $d(D-H)$ & $d(H \ldots A)$ & $d(D \ldots A)$ & $<(\mathrm{DHA})$ \\
\hline \multirow{4}{*}{$\stackrel{+}{+} \underbrace{1}_{\vdots}$} & \multirow{4}{*}{120} & $\mathrm{C}_{\mathrm{ar}}-\mathrm{H}^{\cdots} \mathrm{O}_{\text {diox }}$ & C16-H16...O2 & 0.95 & 2.33 & $3.223(3)$ & 164.0 \\
\hline & & $\mathrm{C}_{\mathrm{ar}}-\mathrm{H}^{\cdots} \mathrm{N}_{\text {cyano }}$ & C18-H18...N2 ${ }^{i i}$ & 0.95 & 2.49 & $3.425(3)$ & 168.1 \\
\hline & & $\mathrm{C}_{\mathrm{ar}}-\mathrm{H}^{\cdots} \mathrm{O}_{\text {diox }}$ Intra & $\mathrm{C} 15-\mathrm{H} 15 \ldots \mathrm{O} 1$ & 0.95 & 2.33 & $2.819(3)$ & 111.5 \\
\hline & & $\mathrm{C}_{\mathrm{ar}}-\mathrm{H}^{\cdots} \mathrm{O}_{\text {diox }}$ Intra & $\mathrm{C} 19-\mathrm{H} 19 . . . \mathrm{O} 1^{\text {iii }}$ & 0.95 & 2.41 & $2.866(3)$ & 108.9 \\
\hline \multirow{4}{*}{\begin{tabular}{l}
\multirow{2}{*}{0} \\
0 \\
$\vdots$ \\
$\vdots$ \\
$\Xi$
\end{tabular}} & \multirow{4}{*}{270} & $\mathrm{C}_{\mathrm{ar}}-\mathrm{H}^{\cdots} \mathrm{O}_{\text {diox }}$ & $\mathrm{C} 16-\mathrm{H} 16 \ldots \mathrm{O} 2^{\mathrm{i}}$ & 0.93 & 2.45 & $3.318(4)$ & 156.3 \\
\hline & & $\mathrm{C}_{\mathrm{ar}}-\mathrm{H}^{\prime \cdots} \mathrm{ONO}$ & C18-H18...N2 ${ }^{i i}$ & 0.93 & 2.60 & $3.508(5)$ & 165.9 \\
\hline & & $\mathrm{C}_{\mathrm{ar}}-\mathrm{H}^{\cdots} \mathrm{O}_{\text {diox }}$ Intra & $\mathrm{C} 15-\mathrm{H} 15 \ldots \mathrm{O} 1$ & 0.93 & 2.50 & $3.046(4)$ & 117.8 \\
\hline & & $\mathrm{C}_{\mathrm{ar}}-\mathrm{H}^{\cdots} \mathrm{O}_{\text {diox }}$ Intra & $\mathrm{C} 19-\mathrm{H} 19 . . .01^{\mathrm{iii}}$ & 0.93 & 2.58 & $3.088(4)$ & 115.1 \\
\hline
\end{tabular}

$\mathrm{P} 2{ }_{1} / \mathrm{c}$ space group $i=\mathrm{x}, \mathrm{y}, \mathrm{z}+1, i i=\mathrm{x},-\mathrm{y}+1 / 2, \mathrm{z}-1 / 2, i i i=-\mathrm{x},-\mathrm{y}+1,-\mathrm{z}+1$ 


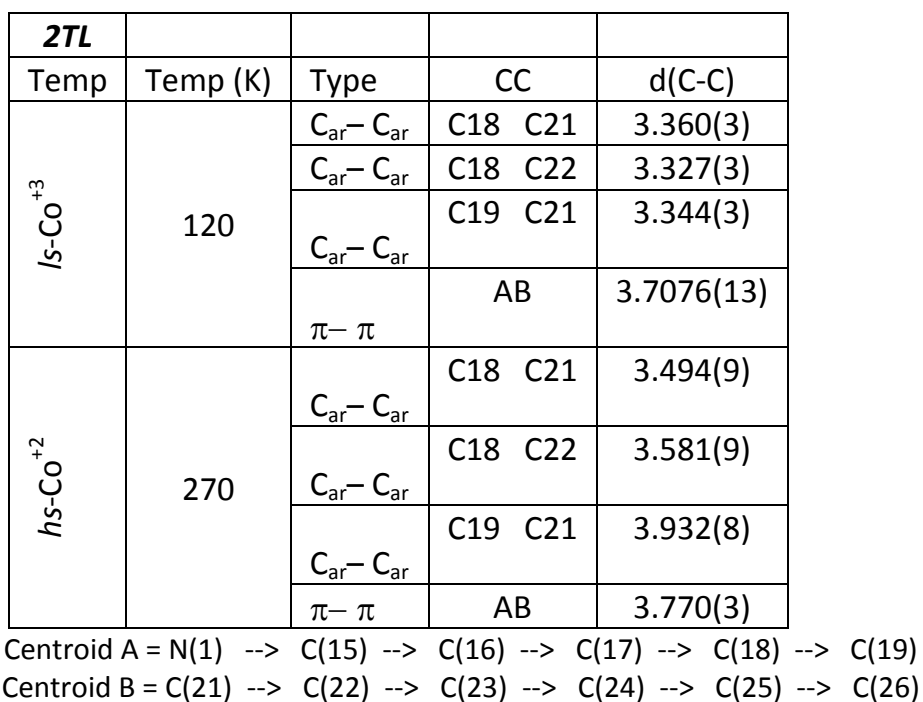

Table S7: Intra and intermolecular hydrogen bonding in [Co(diox $\left.)_{2}(4-\mathrm{CN}-\mathrm{py})_{2}\right]$. Benzene (2BZ) solvate crystal at different temperatures.

\begin{tabular}{|c|c|c|c|c|c|c|c|}
\hline \multicolumn{8}{|l|}{$2 B Z$} \\
\hline Temp & Temp (K) & Type & $D-H \ldots A$ & $d(D-H)$ & $d(H \ldots A)$ & $d(D \ldots A)$ & $<(\mathrm{DHA})$ \\
\hline \multirow{4}{*}{\begin{tabular}{l}
+9 \\
\multirow{1}{+}{0} \\
$\vdots$ \\
$\vdots$ \\
$\vdots$
\end{tabular}} & \multirow{4}{*}{123} & $\mathrm{C}_{\mathrm{ar}}-\mathrm{H}^{\prime \prime} \mathrm{O}_{\text {diox }}$ & $\mathrm{C} 16-\mathrm{H} 16 \ldots \mathrm{OO} 2^{i}$ & 0.95 & 2.31 & $3.228(3)$ & 161.0 \\
\hline & & $\mathrm{C}_{\mathrm{ar}}-\mathrm{H}^{\prime} \mathrm{N}_{\text {cyano }}$ & $\mathrm{C} 18-\mathrm{H} 18 \ldots \mathrm{N} 2 \mathrm{ii}$ & 0.95 & 2.50 & $3.432(3)$ & 168.3 \\
\hline & & $\mathrm{C}_{\mathrm{ar}}-\mathrm{H}^{\cdots} \mathrm{O}_{\text {diox }}$ Intra & C15-H15...O1 & 0.95 & 2.33 & $2.821(2)$ & 111.5 \\
\hline & & $\mathrm{C}_{\mathrm{ar}}-\mathrm{H}^{\prime \prime} \mathrm{O}_{\text {diox }}$ Intra & $\mathrm{C} 19-\mathrm{H} 19 \ldots \mathrm{O} 1^{\mathrm{iii}}$ & 0.95 & 2.42 & $2.861(2)$ & 108.1 \\
\hline \multirow{4}{*}{$\begin{array}{l}+0 \\
\vdots \\
\vdots \\
\vdots \\
\vdots\end{array}$} & \multirow{4}{*}{273} & $\mathrm{C}_{\mathrm{ar}}-\mathrm{H}^{\prime \prime} \mathrm{O}_{\text {diox }}$ & $\mathrm{C} 16-\mathrm{H} 16 \ldots \mathrm{OO} 2^{i}$ & 0.93 & 2.36 & $3.238(4)$ & 157.3 \\
\hline & & $\mathrm{C}_{\mathrm{ar}}-\mathrm{H}^{\prime \cdots} \mathrm{ONO}$ & $\mathrm{C} 18-\mathrm{H} 18 \ldots \mathrm{N} 22^{i \prime}$ & 0.93 & 2.57 & $3.474(5)$ & 163.2 \\
\hline & & $\mathrm{C}_{\mathrm{ar}}-\mathrm{H}^{\prime \prime} \mathrm{O}_{\text {diox }}$ Intra & $\mathrm{C} 15-\mathrm{H} 15 \ldots \mathrm{O} 1$ & 0.93 & 2.47 & $3.023(4)$ & 118.1 \\
\hline & & $\mathrm{C}_{\mathrm{ar}}-\mathrm{H}^{\prime \prime} \mathrm{O}_{\text {diox }}$ Intra & $\mathrm{C} 19-\mathrm{H} 19 . . . \mathrm{O} 1^{\mathrm{iii}}$ & 0.93 & 2.58 & $3.081(4)$ & 114.4 \\
\hline
\end{tabular}

$\mathrm{P} 2{ }_{1} / \mathrm{c}$ space group $i=\mathrm{x}, \mathrm{y}, \mathrm{z}+1, i i=\mathrm{x},-\mathrm{y}+1 / 2, \mathrm{z}-1 / 2, i i i=-\mathrm{x},-\mathrm{y}+1,-\mathrm{z}+1^{\prime}$
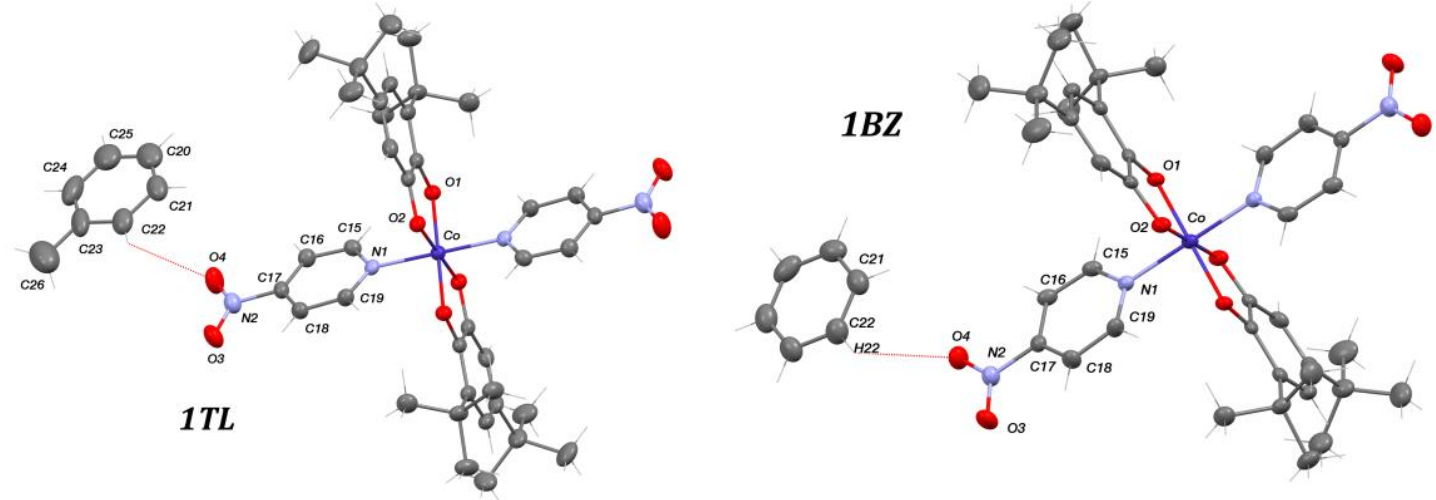

Figure S1. Molecular geometry of $\mathbf{I T L}$ and $\mathbf{I B Z}$ structures. H-bonds between $\mathrm{NO}_{2}$ and solvent molecules are indicated. 

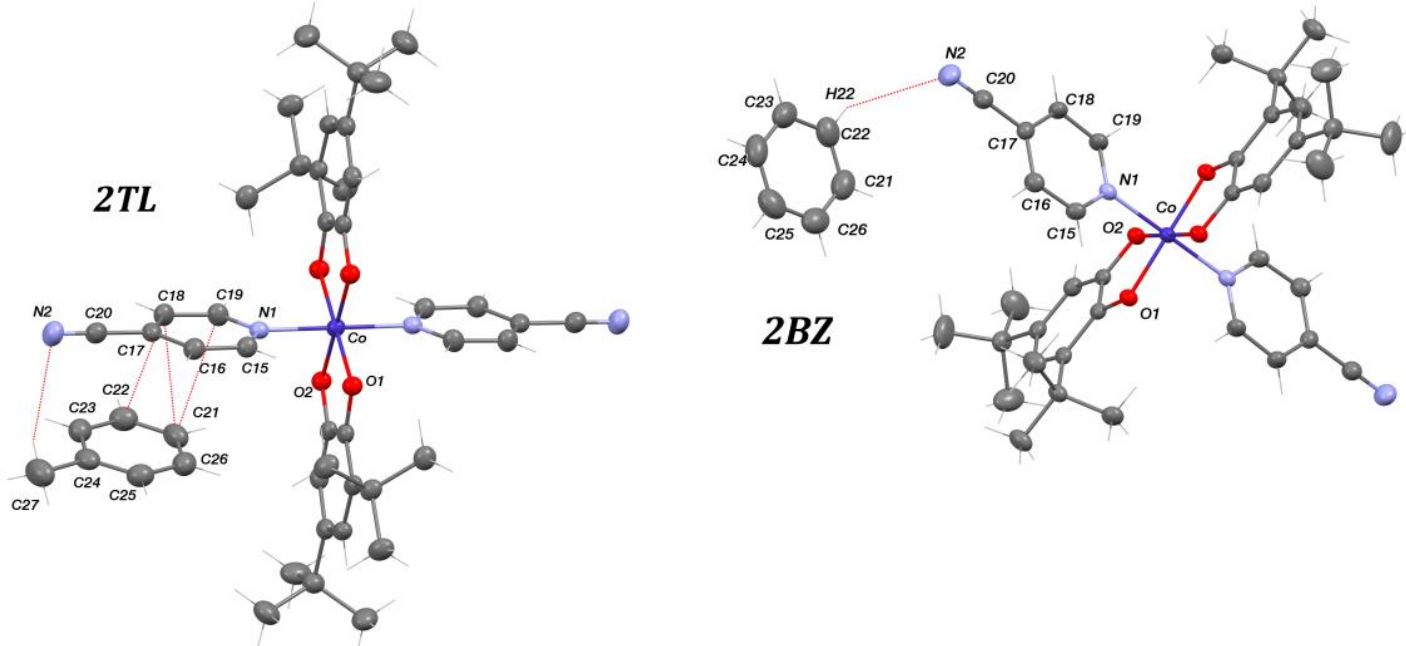

Figure S2. Molecular geometry of $2 T L$ and $2 B Z$ structures. H-bonds between $\mathrm{CN}$ and solvent molecules are indicated.

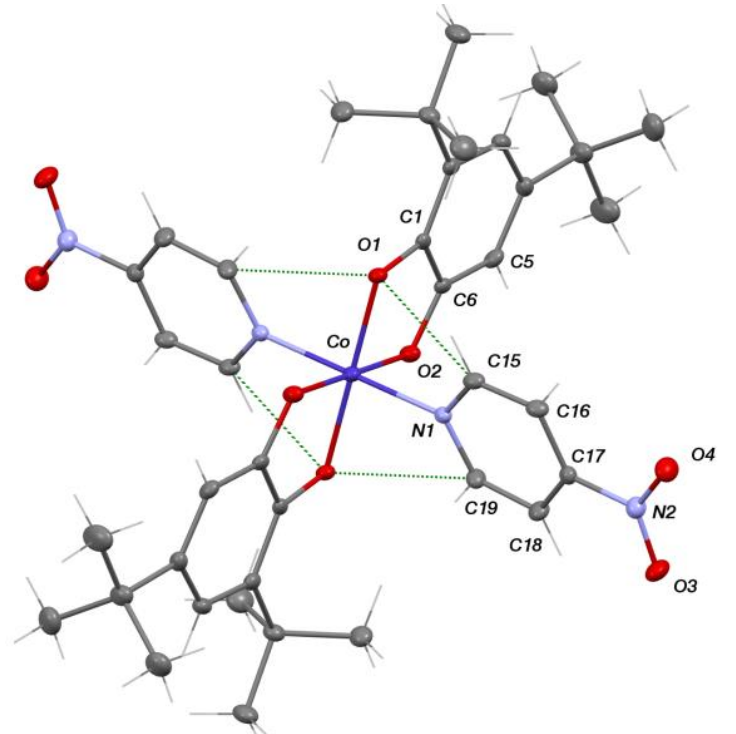

Figure S3. Example of a intramolecular $\mathrm{C}_{\mathrm{ar}}-\mathrm{H}^{\cdots} \mathrm{O}_{\text {diox }} / \mathrm{C} 15-\mathrm{H} 15 \ldots \mathrm{O} 1$ and $\mathrm{C}_{\mathrm{ar}}-\mathrm{H}^{\cdots \cdots} \mathrm{O}_{\text {diox }} / \mathrm{C} 19-\mathrm{H} 19 \ldots \mathrm{O} 1$ hydrogen bonds found in all solvate tautomers.

Refined Co-L distances and converted molar fraction.

\begin{tabular}{|c|c|c|c|c|c|c|c|c|c|}
\hline \multirow[b]{2}{*}{ Crystal } & \multirow[b]{2}{*}{ Temp. } & \multicolumn{3}{|c|}{ X-ray refinements } & \multirow[b]{2}{*}{$\% \mathrm{Co}-\mathrm{O} 1$} & \multirow[b]{2}{*}{$\% \mathrm{Co}-\mathrm{O} 2$} & \multirow[b]{2}{*}{$\% \mathrm{Co}-\mathrm{N}$} & \multirow[b]{2}{*}{ Average\% } & \multirow[b]{2}{*}{ Std $\%$} \\
\hline & & Co-O1 & $\mathrm{Co}-\mathrm{O} 2$ & Co-N & & & & & \\
\hline $2 T L$ & 123 & 1.88 & 1.884 & 1.959 & 0.03 & 0.00 & 0.02 & 0.02 & 0.02 \\
\hline $2 T L$ & 153 & 1.875 & 1.885 & 1.959 & 0.00 & 0.01 & 0.02 & 0.01 & 0.01 \\
\hline $2 T L$ & 183 & 1.894 & 1.902 & 1.98 & 0.12 & 0.10 & 0.11 & 0.11 & 0.01 \\
\hline $2 T L$ & 213 & 1.942 & 1.945 & 2.048 & 0.41 & 0.34 & 0.39 & 0.38 & 0.03 \\
\hline $2 T L$ & 243 & 2.005 & 2.005 & 2.138 & 0.79 & 0.68 & 0.77 & 0.74 & 0.06 \\
\hline $2 T L$ & 270 & 2.029 & 2.025 & 2.171 & 0.94 & 0.79 & 0.90 & 0.88 & 0.08 \\
\hline $2 B Z$ & 123 & 1.88 & 1.8864 & 1.9535 & 0.03 & 0.01 & 0.00 & 0.01 & 0.02 \\
\hline
\end{tabular}




\begin{tabular}{|c|c|c|c|c|c|c|c|c|c|}
\hline $2 B Z$ & 153 & 1.88 & 1.887 & 1.955 & 0.03 & 0.02 & 0.01 & 0.02 & 0.01 \\
\hline $2 B Z$ & 183 & 1.882 & 1.885 & 1.958 & 0.04 & 0.01 & 0.02 & 0.02 & 0.02 \\
\hline $2 B Z$ & 213 & 1.904 & 1.905 & 1.99 & 0.18 & 0.12 & 0.15 & 0.15 & 0.03 \\
\hline $2 \mathrm{BZ}$ & 243 & 1.976 & 1.971 & 2.084 & 0.61 & 0.49 & 0.54 & 0.55 & 0.06 \\
\hline $2 B Z$ & 273 & 2.027 & 2.015 & 2.153 & 0.92 & 0.73 & 0.83 & 0.83 & 0.10 \\
\hline $1 \mathrm{TL}$ & 100 & 1.893 & 1.863 & 1.957 & 0.11 & -0.12 & 0.01 & 0.00 & 0.11 \\
\hline $1 T L$ & 120 & 1.901 & 1.889 & 1.958 & 0.16 & 0.03 & 0.02 & 0.07 & 0.08 \\
\hline $1 \mathrm{TL}$ & 150 & 1.88 & 1.885 & 1.956 & 0.03 & 0.01 & 0.01 & 0.02 & 0.01 \\
\hline $1 \mathrm{TL}$ & 180 & 1.907 & 1.904 & 2.009 & 0.19 & 0.11 & 0.23 & 0.18 & 0.06 \\
\hline $1 T L$ & 210 & 1.96 & 1.992 & 2.074 & 0.52 & 0.60 & 0.50 & 0.54 & 0.06 \\
\hline $1 \mathrm{TL}$ & 240 & 2.006 & 2.046 & 2.17 & 0.80 & 0.91 & 0.90 & 0.87 & 0.06 \\
\hline $1 \mathrm{TL}$ & 270 & 2.039 & 2.06 & 2.184 & 1.00 & 0.98 & 0.96 & 0.98 & 0.02 \\
\hline $\mathrm{NO} 2 \mathrm{BZC}$ & 110 & 1.8812 & 1.8899 & 1.9563 & 0.04 & 0.03 & 0.01 & 0.03 & 0.01 \\
\hline $\mathrm{NO} 2 \mathrm{BZC}$ & 120 & 1.8879 & 1.8923 & 1.962 & 0.08 & 0.05 & 0.04 & 0.05 & 0.02 \\
\hline $\mathrm{NO} 2 \mathrm{BZC}$ & 140 & 2.0375 & 2.0635 & 2.1745 & 0.99 & 1.00 & 0.92 & 0.97 & 0.04 \\
\hline NO2 BZC & 150 & 2.0401 & 2.0702 & 2.1798 & 1.00 & 1.04 & 0.94 & 1.00 & 0.05 \\
\hline $\mathrm{NO} 2 \mathrm{BZC}$ & 170 & 2.0385 & 2.0627 & 2.1792 & 0.99 & 1.00 & 0.94 & 0.98 & 0.03 \\
\hline $\mathrm{NO} 2 \mathrm{BZC}$ & 200 & 2.0391 & 2.0636 & 2.183 & 1.00 & 1.00 & 0.95 & 0.99 & 0.03 \\
\hline $\mathrm{NO} 2 \mathrm{BZC}$ & 230 & 2.0391 & 2.0628 & 2.1845 & 1.00 & 1.00 & 0.96 & 0.99 & 0.02 \\
\hline $\mathrm{NO} 2 \mathrm{BZC}$ & 260 & 2.0427 & 2.0605 & 2.1886 & 1.02 & 0.99 & 0.98 & 0.99 & 0.02 \\
\hline $\mathrm{NO} 2 \mathrm{BZC}$ & 280 & 2.0397 & 2.0618 & 2.1912 & 1.00 & 0.99 & 0.99 & 0.99 & 0.01 \\
\hline $\mathrm{NO} 2 \mathrm{BZH}$ & 110 & 1.8812 & 1.8899 & 1.9563 & 0.04 & 0.03 & 0.01 & 0.03 & 0.01 \\
\hline $\mathrm{NO} 2 \mathrm{BZH}$ & 125 & 1.8821 & 1.8881 & 1.9564 & 0.04 & 0.02 & 0.01 & 0.03 & 0.02 \\
\hline $\mathrm{NO} 2 \mathrm{BZH}$ & 140 & 1.8824 & 1.8883 & 1.9556 & 0.04 & 0.02 & 0.01 & 0.03 & 0.02 \\
\hline $\mathrm{NO} 2 \mathrm{BZH}$ & 155 & 1.8838 & 1.8881 & 1.957 & 0.05 & 0.02 & 0.01 & 0.03 & 0.02 \\
\hline $\mathrm{NO} 2 \mathrm{BZH}$ & 170 & 2.0373 & 2.0611 & 2.178 & 0.99 & 0.99 & 0.93 & 0.97 & 0.03 \\
\hline $\mathrm{NO} 2 \mathrm{BZH}$ & 185 & 2.0368 & 2.0614 & 2.177 & 0.98 & 0.99 & 0.93 & 0.97 & 0.03 \\
\hline $\mathrm{NO} 2 \mathrm{BZH}$ & 200 & 2.0373 & 2.0619 & 2.181 & 0.99 & 0.99 & 0.95 & 0.98 & 0.03 \\
\hline $\mathrm{NO} 2 \mathrm{BZH}$ & 215 & 2.041 & 2.065 & 2.185 & 1.01 & 1.01 & 0.96 & 0.99 & 0.03 \\
\hline $\mathrm{NO} 2 \mathrm{BZH}$ & 250 & 2.0382 & 2.0634 & 2.19 & 0.99 & 1.00 & 0.98 & 0.99 & 0.01 \\
\hline $\mathrm{NO} 2 \mathrm{BZH}$ & 290 & 2.0395 & 2.0639 & 2.194 & 1.00 & 1.01 & 1.00 & 1.00 & 0.00 \\
\hline Experimental & \multicolumn{3}{|c|}{ Co3+ } & \multicolumn{3}{|c|}{$\mathrm{Co} 2+$} & & & \\
\hline & Co-O1 & $\mathrm{Co}-\mathrm{O} 2$ & $\mathrm{CO}-\mathrm{N}$ & $\mathrm{Co}-\mathrm{O} 1$ & $\mathrm{Co}-\mathrm{O} 2$ & $\mathrm{CO}-\mathrm{N}$ & & & \\
\hline Ave & 1.884 & 1.884 & 1.958 & 2.034 & 2.038 & 2.173 & & & \\
\hline \multirow[t]{2}{*}{ STD } & 0.006 & 0.010 & 0.004 & 0.006 & 0.021 & 0.015 & & & \\
\hline & \multicolumn{2}{|c|}{$1.884(8)$} & & \multicolumn{2}{|c|}{$2.036(14)$} & & & & \\
\hline
\end{tabular}

\section{$\left(h s-\mathrm{Co}^{2+}\right)$ molar fraction fitting data.}


QTIPLOT output: http://soft.proindependent.com/qtiplot.html

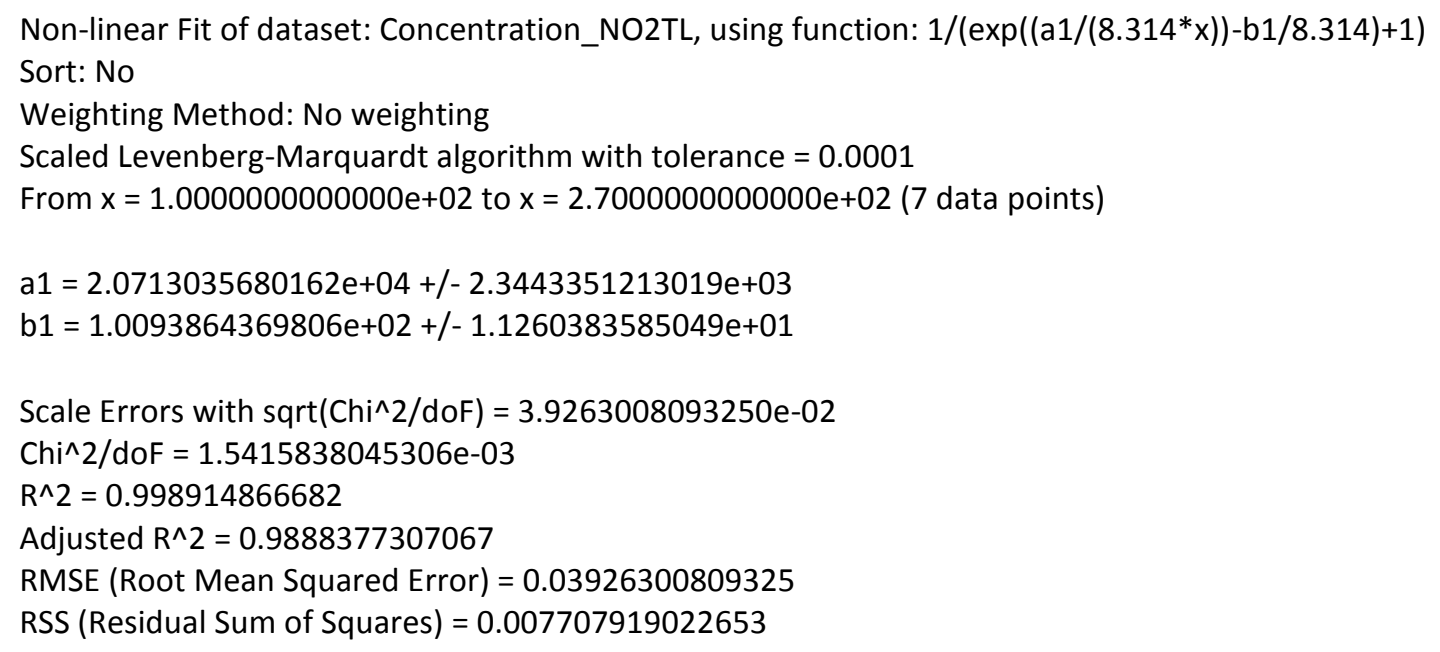

Non-linear Fit of dataset: Concentration_CnTL, using function: 1/(exp((a1/(8.314*x))-b1/8.314)+1) Sort: No

Weighting Method: No weighting

Scaled Levenberg-Marquardt algorithm with tolerance $=0.0001$

From $x=1.2300000000000 e+02$ to $x=2.7000000000000 e+02$ ( 6 data points)

$\mathrm{a} 1=2.0271192706210 \mathrm{e}+04+/-9.5509763009667 \mathrm{e}+02$

b1 $=9.1648720192934 \mathrm{e}+01+/-4.2675328770183 \mathrm{e}+00$

Scale Errors with sqrt $\left(\mathrm{Chi}^{\wedge} 2 / \mathrm{doF}\right)=1.6762913609874 \mathrm{e}-02$

$\mathrm{Chi}^{\wedge} 2 / \mathrm{doF}=2.8099527269211 \mathrm{e}-04$

$\mathrm{R}^{\wedge} 2=0.999141435007$

Adjusted $R^{\wedge} 2=0.9973826818863$

RMSE (Root Mean Squared Error) $=0.01676291360987$

RSS (Residual Sum of Squares) $=0.001123981090768$

Non-linear Fit of dataset: Concentration_CnBz, using function: 1/(exp $\left.\left(\left(a 1 /\left(8.314^{*} \mathrm{x}\right)\right)-\mathrm{b} 1 / 8.314\right)+1\right)$

\section{Sort: No}

Weighting Method: No weighting

Scaled Levenberg-Marquardt algorithm with tolerance $=0.0001$

From $x=1.2300000000000 \mathrm{e}+02$ to $x=2.7300000000000 \mathrm{e}+02$ ( 6 data points)

a1 $=2.6700721754340 \mathrm{e}+04+/-1.0266172492646 \mathrm{e}+03$

b1 $=1.1133768895177 \mathrm{e}+02+/-4.2571753247401 \mathrm{e}+00$

Scale Errors with sqrt $\left(\mathrm{Chi}^{\wedge} 2 / \mathrm{doF}\right)=1.2394591404468 \mathrm{e}-02$

$\mathrm{Chi}^{\wedge} 2 / \mathrm{doF}=1.5362589608370 \mathrm{e}-04$

$\mathrm{R}^{\wedge} 2=0.9929951122119$

Adjusted $R^{\wedge} 2=0.998289434405$

RMSE (Root Mean Squared Error) $=0.01239459140447$

RSS (Residual Sum of Squares) $=0.0006145035843348$ 
Non-linear Fit of dataset: Concentration_NO2BzH, using function: 1/(exp $((a 1 /(8.314 * x))-b 1 / 8.314)+1)$ Sort: No

Weighting Method: No weighting

Scaled Levenberg-Marquardt algorithm with tolerance $=0.0001$

From $x=1.1000000000000 \mathrm{e}+02$ to $x=2.9000000000000 \mathrm{e}+02$ (10 data points)

a1 $=1.0139656882250 \mathrm{e}+05+/-1.6178329923933 \mathrm{e}+04$

b1 $=6.2529840828935 e+02+/-9.9864636173397 e+01$

Scale Errors with sqrt $\left(\mathrm{Chi}^{\wedge} 2 / \mathrm{doF}\right)=2.2896669558641 \mathrm{e}-02$

Chi^2/doF $=5.2425747687758 \mathrm{e}-04$

$\mathrm{R}^{\wedge} 2=0.9992432983594$

Adjusted $\mathrm{R}^{\wedge} 2=0.9975286611032$

RMSE (Root Mean Squared Error) $=0.02289666955864$

RSS (Residual Sum of Squares) $=0.004194059815021$

Non-linear Fit of dataset: Concentration_NO2BzC, using function: 1/(exp $\left.\left(\left(a 1 /\left(8.314^{*} x\right)\right)-b 1 / 8.314\right)+1\right)$ Sort: No

Weighting Method: No weighting

Scaled Levenberg-Marquardt algorithm with tolerance $=0.0001$

From $x=1.1000000000000 e+02$ to $x=2.8000000000000 e+02$ (9 data points)

$\mathrm{a} 1=4.4629303500985 \mathrm{e}+04+/-4.2411656953471 \mathrm{e}+03$

b1 $=3.4758199206358 \mathrm{e}+02+/-3.4041138940904 \mathrm{e}+01$

Scale Errors with sqrt $\left(\mathrm{Chi}^{\wedge} 2 / \mathrm{doF}\right)=1.5358072385517 \mathrm{e}-02$

$\mathrm{Chi}^{\wedge} 2 / \mathrm{doF}=2.3587038739877 \mathrm{e}-04$

$\mathrm{R}^{\wedge} 2=0.9944793589565$

Adjusted $\mathrm{R}^{\wedge} 2=0.9984232510034$

RMSE (Root Mean Squared Error) $=0.01535807238552$

RSS (Residual Sum of Squares) $=0.001651092711791$ 